\title{
Juristische Antworten auf die geschlechterspezifische Gewalt in Spanien*
}

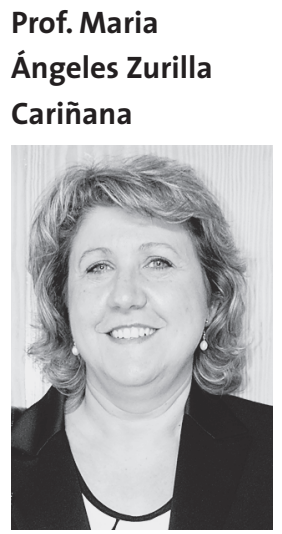

Ordentliche Universitätsprofessorin für Zivilrecht und Dekanin an der Universität Castilla La Mancha, Mitglied der spanischen Juristinnenvereinigung THEMIS

\section{Problemstellung}

Gewalt gegen Frauen gab es schon immer. Wirklich neu ist, dass sie heute als Gewalt anerkannt und nicht mehr einfach hingenommen wird. Eine wichtige Rolle bei dieser Entwicklung haben Menschenrechtsorganisationen, Frauenrechtsgruppierungen und internationale Organisationen gespielt. Die Kommunikationsmedien haben gleichfalls einen entscheidenden Anteil an der Bewusstwerdung des Problems in der Gesellschaft.

In Spanien wird die Gewalt gegen Frauen als Gewalt im Geschlechterverhältnis bezeichnet, um die Bedeutung zu unterstreichen, die kulturelle und gesellschaftliche Faktoren hierbei spielen. Der Begriff umfasst alle Formen von psychischer Gewalt, von Misshandlung und sexueller Ausbeutung, denen Frauen aufgrund ihres Geschlechts ausgesetzt sind.

1993 hat die UNO in Wien klargestellt, dass Frauenrechte zu den Menschenrechten gehören und dass Gewalt gegen Frauen einen Verstoß gegen Menschenrechte darstellt. Gewalt gegen Frauen wurde dabei definiert als jeglicher Akt der Gewaltanwendung oder Zwangsausübung mit dem Ziel der Herstellung oder Aufrechterhaltung von Macht und Unterordnungsverhältnissen zwischen Männern und Frauen.

Ausgehend von der Konferenz von Peking 1995 wurde das Phänomen der Gewalt im Geschlechterverhältnis international als gesellschaftliches Problem erkannt und klar in den Kontext von Menschenrechten und Gleichheit zwischen Frau und Mann gestellt. Die Weltfrauenkonferenz versteht unter Gewalt gegen Frauen „jeden Akt von geschlechtsbezogener Gewalt, der als mögliche oder tatsächliche Folge einen physischen, sexuellen oder psychischen Schaden nach sich zieht, einschließlich Drohungen, Nötigung, Freiheitsberaubung, sowohl im öffentlichen oder privaten Raum“. Seitdem wurde in Europa eine Entwicklung angestoßen, die einen anderen justiziellen Umgang mit den Delikten nach sich zog, die zuvor als Vergehen gegen die Familienehre behandelt wurden oder die ganz einfach - da als Privatangelegenheiten angesehen - keiner Bestrafung zugeführt wurden.

\section{Gesetzeslage in Spanien}

In den letzten Jahren wurden in Spanien verschiedene effektive Gesetzgebungsvorhaben umgesetzt, so das Gesetz „Ley Orgánica“ 11/2003 vom 29.
September, über Maßnahmen auf dem Gebiet der inneren Sicherheit, häuslichen Gewalt und Integration von Ausländern; das Gesetz „Ley Orgánica“ 15/2003 vom 25. November zum Gesetz 10/1995 vom 23. November, Änderungsgesetz zum Strafgesetzbuch; oder das Gesetz 27/2003 vom 31. Juli zum Schutz von Opfern von häuslicher Gewalt. Neben diesen wichtigen Gesetzen sind auch die von verschiedenen Autonomen Regionen im Rahmen ihrer Zuständigkeiten erlassenen Normen zu erwähnen.

Der gesetzgeberische Höhepunkt wurde im Jahr 2004 erreicht, mit dem Gesetz „Ley Orgánica“ $1 / 2004$ vom 28. Dezember über umfassende Schutzmaßnahmen gegen Gewalt im Geschlechterverhältnis. Das Gesetz befolgt die Empfehlungen der internationalen Organisationen und unternimmt eine globale Reaktion auf die Gewalt, die gegenüber Frauen ausgeübt wird.

Die Darstellung der Gesetzeslage wäre nicht abschließend, wenn nicht auch das spanische Zivilgesetzbuch kurz erwähnt würde, ein Gesetzeswerk, dessen Ursprünge auf das Jahr 1889 zurückgehen. Die letzte uns hier interessierende Reform fand im Jahr 2005 statt, mit dem Erlass des Gesetzes 15/2005 vom 8. Juli, Änderungsgesetz zum Zivilgesetzbuch, betreffend eheliche Trennung und Scheidung. Das Gesetz hat den Artikel 81.2 geändert, der jetzt wie folgt lautet:

„Die Trennung wird gerichtlich ausgesprochen auf Antrag eines der Ehegatten, frühestens drei Monate nach der Eheschließung. Diese Mindestfrist für eine Antragstellung gilt nicht, wenn eine Gefahr für Leben, körperliche, seelische oder sexuelle Unversehrtheit oder die Freiheit des antragstellenden Ehegatten oder der Kinder von beiden oder von einem der Ehegatten glaubhaft gemacht wird.“

Artikel 86 des Zivilgesetzbuchs bestimmt:

„Die Scheidung wird, unabhängig von der Form der Eheschließung, auf Antrag beider Ehegatten oder eines Ehegatten mit Einverständnis des anderen gerichtlich ausgesprochen, wenn die Voraussetzungen und Bedingungen des Artikels 81 vorliegen."

\footnotetext{
* Dies ist eine gekürzte Fassung eines wissenschaftlichen Artikels mit ausführlichen Nachweisen, der der Kommission Gewalt gegen Frauen und Kinder vorliegt. Übersetzung durch Staatsanwältin Sabine KräuterStockton, Redaktion durch Nancy Gage-Lindner; Mitglieder der Kommission Gewalt gegen Frauen und Kinder des djb.
} 
Auch wenn in Spanien die nach der Eheschließung einzuhaltende Mindestfrist für Trennung und Scheidung für sich gesehen mit drei Monaten sehr kurz ist, hat das geschärfte Problembewusstsein betreffend die Gewalt im Geschlechterverhältnis den Gesetzgeber zu der Klarstellung veranlasst, dass unter Umständen keine auch noch so kurze Zeit bis zur Trennung oder Scheidung zumutbar ist, und zwar wenn gleich welche Form von Gewalt glaubhaft gemacht wird, sei sie gegen den Ehegatten oder auch gegen die Kinder gerichtet.

Eine andere Stelle, an der sich im Zivilrecht das Vorkommen von Gewalt auswirken kann, ist in Art. 170 Zivilgesetzbuch geregelt. Hier ist die Entziehung der elterlichen Sorge für den Fall vorgesehen, dass ein Elternteil seine elterlichen Pflichten nicht erfüllt oder sich strafbar macht. Zu der Nichterfüllung der elterlichen Pflichten zählt dabei Misshandlung der Kinder und auch der Mutter der Kinder.

Von all den genannten Gesetzen konzentrieren wir uns aber auf die beiden mit den weitestgehenden Auswirkungen, nicht nur wegen ihres Inhalts, sondern auch weil sie einen echten Meilenstein in der Bekämpfung der Gewalt im Geschlechterverhältnis in Spanien darstellen:

\section{Gesetz zur Regelung der Schutzanordnung für Opfer von Gewalt im Geschlechterverhältnis („Ley ordenadora de la Orden de Protección de las Víctimas de Violencia de Género")}

Dieses Gesetz benennt erstmals in Spanien das Bedürfnis nach einer koordinierten und umfassenden Antwort seitens aller öffentlichen Einrichtungen auf das gravierende Problem der Gewalt im Geschlechterverhältnis. Die Schutzanordnung kombiniert im Rahmen eines beschleunigten und vereinfachten Verfahrens vor dem Einzelrichter verschiedene Instrumente des Schutzes und der Fürsorge für Opfer dieser Straftaten. Das Opfer erhält einen umfassenden Schutz, der gleichzeitig zivilrechtliche und strafrechtliche Elemente enthält, denn durch ein einzelnes justizielles Verfahren werden sowohl freiheitsentziehende oder -beschränkende Maßnahmen gegen den Täter getroffen, die eine erneute Annäherung an das Opfer verhindern, als auch solche, die Sicherheit und juristischen Schutz für das Opfer und dessen Familie bewirken, unabhängig von dem stark formalisierten und daher schwerfälligen und langsamen $\mathrm{Fa}$ milienprozess.

Die Schutzanordnung bedeutet gleichzeitig, dass die verschiedenen öffentlichen Einrichtungen auf Landes-, Regionalund Lokalebene unverzüglich die in ihren jeweiligen juristischen Systemen vorgehaltenen Schutzinstrumente zur Anwendung bringen. Genau hier zeigt sich das innovativste Element der Regelung.

Das Gesetz weist die Zuständigkeit für den Erlass einer Schutzanordnung dem Einzelrichter (Juez de instrucción) in seiner Funktion als Präventivorgan zu. Die ausgeworfene Entscheidung kann gleichzeitig beinhalten:
- Freiheitsbeschränkende Maßnahmen gegen den Täter im Sinn von Annäherungsverboten an das Opfer.

- Maßnahmen, die auf juristischen Schutz für das Opfer und seine Familie abzielen, außerhalb des formalisierten Ehescheidungsprozesses.

Das Gesetz über die Schutzanordnung modifiziert außerdem das Strafprozessrecht, indem es eine zusätzliche Vorschrift einfügt:

- Die Schutzanordnung wird erlassen, wenn hinreichende Anhaltspunkte für die Begehung einer Straftat gegen das Leben, die körperliche oder psychische Unversehrtheit oder die sexuelle Selbstbestimmung vorliegen und infolgedessen eine Gefahrensituation für das Opfer anzunehmen ist.

- Die Schutzanordnung wird auf Antrag des Opfers oder der Staatsanwaltschaft vom Bereitschaftsgericht erlassen. Unbeschadet der allgemeinen, gesetzlich festgelegten Anzeigepflicht sind die öffentlichen und privaten Hilfeeinrichtungen, die Kenntnis von einer der genannten Tatsachen erhalten, dazu verpflichtet, diese Tatsachen unverzüglich dem diensthabenden Richter/der diensthabenden Richterin oder der Staatsanwaltschaft zur Kenntnis zu bringen, mit dem Ziel der Einleitung eines Verfahrens zum Erlass einer Schutzanordnung.

- Ein Antrag auf Erlass einer Schutzanordnung kann direkt beim Gericht oder der Staatsanwaltschaft gestellt werden oder bei der Polizei, bei Opferschutzstellen oder Sozialdiensten oder Beratungsstellen in der öffentlichen Verwaltung. Der Antrag muss dann unverzüglich der zuständigen Richterin bzw. dem zuständigen Richter vorgelegt werden.

- Sobald der Antrag bei Gericht eingegangen ist, muss die zuständige Richterin oder der zuständige Richter unverzüglich einen Anhörungstermin bestimmen, zu dem das Opfer, seine anwaltliche Vertretung, der Täter sowie seine Rechtsanwältin oder sein Rechtsanwalt als auch die Staatsanwaltschaft geladen werden. Der Termin muss innerhalb einer Frist von maximal 72 Stunden nach Eingang des Antrags stattfinden. Um ein Zusammentreffen des Täters mit dem Opfer sowie dessen Kindern und restlichen Familienangehörigen zu vermeiden, wird angeordnet, dass die Anhörung getrennt stattfindet.

- Im Anschluss an die Anhörung entscheidet die Richterin bzw. der Richter über den Antrag auf Schutzanordnung und beschließt die konkreten Maßnahmen.

- Die Schutzanordnung gewährt dem Opfer einen umfassenden Schutzstatus durch Maßnahmen zivil- und strafrechtlicher Art. Die zivilrechtlichen Maßnahmen müssen vom Opfer oder seiner anwaltlichen Vertretung beantragt werden oder durch die Staatsanwaltschaft, wenn es minderjährige Kinder gibt, falls nicht schon zuvor Schutz durch einen zivil- oder familienrechtlichen Gerichtsbeschluss gewährt wurde. An Maßnahmen kommt die Zuweisung der Familienwohnung in Betracht, die Regelung des Sorge- und Besuchsrechts betreffend die gemeinsamen Kinder sowie des Unterhalts ebenso wie alle anderen Anordnungen, die zum Schutz von Minderjährigen geeignet sind. 
- Die zivilrechtlichen Maßnahmen im Rahmen der Schutzanordnung haben Geltung für einen Zeitraum von 30 Tagen. Wenn innerhalb dieser Frist durch das Opfer oder seine Vertretung eine Klage vor dem Zivil- oder Familiengericht eingereicht wird, verlängert sich die Geltungsfrist der getroffenen Anordnungen um weitere 30 Tage. Innerhalb dieser Frist wird dann durch das Familiengericht eine Bestätigung, Änderung oder Aufhebung beschlossen.

- Die Schutzanordnung muss den Parteien bekannt gegeben und dem Opfer und insbesondere der zuständigen öffentlichen Verwaltungsbehörde unverzüglich mitgeteilt werden, damit von dort aus angemessene Schutzmaßnahmen auf sozialer oder juristischer Ebene, im Gesundheitsoder sonstigem Bereich eingeleitet werden können. $\mathrm{Zu}$ diesem Zweck wird eine umfassende und systematische gegenseitige Abstimmung und Koordination der verschiedenen Behörden und Einrichtungen untereinander eingerichtet. Die Schutzanordnung bedeutet, dass das Opfer laufend über das Prozessverhalten des Verdächtigen und die prozessrechtliche und sonstige Situation informiert wird, ebenso über die Anordnung oder Fortdauer aller Schutzmaßnahmen. Das Opfer muss auch immer über den Stand der freiheitsentziehenden Maßnahmen, die gegenüber dem Verdächtigen getroffen wurden, informiert sein, daher muss auch die Strafvollzugsbehörde von der Schutzanordnung in Kenntnis gesetzt werden.

- Die Schutzanordnung wird in ein zentrales Register für den Schutz von Opfern häuslicher Gewalt eingetragen.

\section{Gesetz zum umfassenden Schutz gegen Gewalt im Ge- schlechterverhältnis (Ley Orgánica de Medidas de Protección Integral contra la Violencia de Género")}

Mit derselben Zielsetzung wie das „Gesetz zur Regelung der Schutzanordnung“ bietet das „Gesetz zum umfassenden Schutz gegen Gewalt im Geschlechterverhältnis“ in Anlehnung an die Empfehlungen der internationalen Organisationen eine globale Antwort auf Gewalt gegen Frauen. Das Gesetz enthält sowohl Regelungen der Prävention im sozialen Bereich und im Erziehungswesen, der Beratung und Nachsorge für die Opfer, als auch einen zivilrechtlichen Normenkatalog, betreffend Familien und sonstige nichteheliche Lebensgemeinschaften, in denen sich Gewalttaten ereignen. Das Problem der Gewalt im Geschlechterverhältnis wird in dem Gesetz in einer umfassenden und multidisziplinären Weise angegangen, angefangen vom Prozess der Sozialisation und Erziehung. Es umfasst eine Einleitung, fünf große Abschnitte und 20 ergänzende Vorschriften, zwei Übergangsvorschriften, eine Aufhebungsvorschrift und sieben abschließende Vorschriften.

Die Einleitung fasst die allgemeinen Vorschriften des Gesetzes betreffend Ziel, Zweck sowie Grundlagen zusammen. Der erste Artikel legt als Ziel den Kampf gegen die Gewalt fest, die als Ergebnis der ungleichen Strukturen und Machtverhältnisse von Männern gegenüber Frauen zu sehen ist.
Der zweite Artikel zeigt die Grundprinzipien auf, unter anderem Ausbau der aktuell zulässigen Maßnahmen auf dem Gebiet des Straf- und Strafprozessrechts, um einen umfassenden Schutz zu sichern; Koordinierung der Präventionsmaßnahmen auf allen Ebenen der öffentlichen Verwaltung; arbeitsrechtliche und wirtschaftliche Stärkung der Rechte von Frauen, die Opfer häuslicher Gewalt wurden, mit dem Ziel, ihre gesellschaftliche Integration zu erleichtern; Einrichtung eines umfassenden Systems von staatlichen Stellen mit der Aufgabe und Kompetenz zur Implementierung eines fortlaufenden Verbesserungsprozesses; Förderung der Spezialisierung von Berufsgruppen, die in den Prozess der Information, Beratung und Schutzgewährung von Gewaltopfern einbezogen sind.

Im ersten Abschnitt werden Maßnahmen zur Sensibilisierung, Prävention, Aufdeckung und Intervention in unterschiedlichen Bereichen des öffentlichen Lebens festgeschrieben (Erziehungs- und Gesundheitswesen, Öffentlichkeit).

Im zweiten Abschnitt wird der Zugang zu Information und umfassender sozialer Unterstützung durch soziale Dienste garantiert, auch der Anspruch auf kostenfreie juristische Beratung und Unterstützung; weiter werden arbeitsrechtliche und sozialhilferechtliche Ansprüche sowie Ansprüche von Mitarbeiterinnen der öffentlichen Verwaltung (z.B. auf Beurlaubung mit Arbeitsplatzgarantie, Versetzung, Freistellung) formuliert und finanzielle Ansprüche für Opfer, die über keine Mittel verfügen. Außerdem sind Frauen, die Opfer von Gewalt im Geschlechterverhältnis wurden, bevorrechtigt, wenn es um Bewerbungen für Sozialwohnungen und öffentliche Einrichtungen für Ältere geht.

Der dritte Abschnitt betrifft die Einrichtung und Organisation von staatlichen Institutionen. Hier werden mit der Spezialstelle Gewalt gegen Frauen in der Regierung und dem Staatlichen Observatorium zwei wichtige Behörden geschaffen.

Die Spezialstelle ist beim Arbeits- und Sozialministerium eingerichtet und hat die Aufgabe, politische Forderungen betreffend die Gewalt im Geschlechterverhältnis zu formulieren, die durch die Regierung umzusetzen sind. Außerdem soll sie Aktionen auf diesem Gebiet in Zusammenarbeit mit den betreffenden Behörden initiieren und koordinieren.

Das Staatliche Observatorium ist ein Kollegialorgan, das ebenso beim Arbeits- und Sozialministerium angesiedelt ist und Erhebungen, Evaluationen, Studien und Untersuchungen in Zusammenarbeit mit anderen Institutionen durchführen und Handlungsempfehlungen auf dem Gebiet der Gewalt im Geschlechterverhältnis entwickeln soll. Das Staatliche Observatorium legt der Staatsregierung und den Regionalregierungen jährliche Berichte über die Entwicklung auf dem Gebiet der Gewalt gegen Frauen vor.

Dieser Abschnitt sieht auch die Einrichtung spezialisierter Sachgebiete bei der Polizei und den Sicherheitsbehörden vor, die präventive Aufgaben wahrnehmen und mit der ordnungsgemäßen Durchführung und Überwachung der getroffenen justiziellen Maßnahmen beauftragt sind. Hinzu 
kommt die Erarbeitung von Plänen zur Koordinierung und Abstimmung des gemeinsamen Handelns der jeweiligen Behörden und Dienste.

Der vierte Abschnitt trifft besondere Regelungen im Straf- und Strafverfahrensrecht. Das spanische Strafgesetzbuch wird - soweit es um Fälle von Gewalt im Geschlechterverhältnis geht - in einer Reihe von Punkten modifiziert, sowohl zum Schutz vor Körperverletzung, als auch vor Drohungen, Nötigungen und Belästigungen. Die Strafzumessung wird dadurch beeinflusst, dass das Opfer der Straftat (ehemalige) Ehefrau oder Lebensgefährtin des Täters ist, auch ohne gemeinsamen Wohnsitz.

Der fünfte Abschnitt enthält für den Rechtsschutz von Opfern von Gewalt im Geschlechterverhältnis eine Reihe wichtiger Regelungen: die Einrichtung von auf Gewalt gegen Frauen spezialisierten Gerichten und von Sonderdezernaten bei der Staatsanwaltschaft für Fälle von Gewalt gegen Frauen.

In Artikel 43 des genannten Abschnitts wird bezüglich das Gerichtswesens bestimmt, dass in allen Amtsgerichtsbezirken Gerichte einzurichten sind, die auf Fälle von Gewalt gegen Frauen spezialisiert sind.

Weiter wird den für Gewalt gegen Frauen zuständigen Gerichten auch die Strafgewalt zugeschrieben für Straftaten wie Tötungsdelikte, Abtreibungen, Körperverletzungen, Freiheitsberaubungen, gegen die sexuelle Selbstbestimmung oder alle anderen Straftaten, die unter Anwendung von Gewalt oder Einschüchterung begangen wurden und sich gegen die Ehefrau oder Lebensgefährtin oder sonstiges Opfer richten, welches mit dem Täter in ähnlich enger emotionaler Bindung lebt oder lebte, auch ohne gemeinsamen Wohnsitz. Sie erhalten auch die Kompetenz, über Straftaten zu erkennen, die gegenüber eigenen Abkömmlingen oder der Ehefrau oder Lebensgefährtin oder deren Abkömmlingen (sofern sie mit ihr zusammenleben) begangen wurden, wenn auch eine Tat im Zusammenhang mit Gewalt im Geschlechterverhältnis begangen wurde. Die für Gewalt gegen Frauen zuständigen Gerichte sind außerdem für Schutzanordnungen zugunsten von Opfern zuständig.

Außerdem treffen die für Gewalt gegen Frauen zuständigen Gerichte auch Entscheidungen in Abstammungsfragen, über Nichtigkeit, Trennung und Scheidung der Ehe, über das Verhältnis zwischen Eltern und Kindern, über Sorgerecht und Aufenthaltsbestimmungsrecht minderjährige Kinder betreffend, sowie über Unterhaltsansprüche, Adoptionen, und über verwaltungsrechtlich erlassene Minderjährigenschutzverfügungen. Die ausschließliche Zuständigkeit des für Gewalt gegen Frauen zuständigen Gerichts besteht, wenn die folgenden Voraussetzungen zusammentreffen: Es handelt sich um einen zivil- oder familienrechtlichen Prozess, der irgendeine der genannten Materien zum Gegenstand hat; eine der Parteien des Zivilprozesses ist Opfer von Gewalt im Geschlechterverhältnis geworden; eine der Parteien ist als Täter oder Mittäter an Gewalthandlungen im Geschlechterverhältnis beschuldigt; vor dem für Gewalt gegen Frauen zuständigen Gericht ist eine Strafsache wegen einer Straftat im Zusammenhang mit Gewalt gegen eine Frau anhängig oder es wurde eine Schutzanordnung für ein Opfer von Gewalt im Geschlechterverhältnis erlassen. Wenn die Richterin oder der Richter feststellt, dass die gegenständlichen Handlungen keine Gewalt im Geschlechterverhältnis darstellen, verweist sie oder er die Sache an das nach allgemeinen Vorschriften zuständige Gericht.

Artikel 57 des Gesetzes regelt, dass die Zivil- oder Familienrichterin oder der Zivil- oder Familienrichter ihre oder seine sachliche Zuständigkeit verliert, wenn Gewalttaten gegen die Frau ausgeführt wurden. Wenn eine oder ein in erster Instanz mit einem Zivilverfahren befasste Richterin oder Richter feststellt, dass Gewalt im Geschlechterverhältnis begangen wurde, die zu einem Strafverfahren oder zu einer Schutzanordnung führte, muss sie oder er sich für unzuständig erklären und die Akten dem für Gewalt gegen Frauen zuständigen Gericht vorlegen, es sei denn, eine mündliche Verhandlung hat schon stattgefunden. Wenn die Richterin oder der Richter Kenntnis von einer Gewalttat erhält, die bisher nicht zu einem Strafverfahren oder einer Schutzanordnung geführt hat, lädt sie oder er die Parteien zu einem Termin zur Staatsanwaltschaft vor, der innerhalb der nächsten 24 Stunden stattfindet. Binnen weiterer 24 Stunden wird danach die Staatsanwaltschaft entscheiden, ob ein Verfahren wegen Gewalt im Geschlechterverhältnis eingeleitet wird oder eine Schutzanordnung vor dem für Gewalt zuständigen Gericht beantragt wird. Wenn ein Verfahren eingeleitet oder eine Schutzanordnung beantragt wird, bleibt die Zivilrichterin bzw. der Zivilrichter noch so lange zuständig, bis das für Gewalt gegen Frauen zuständige Gericht die Sache übernommen hat.

Wenn das für Gewalt gegen Frauen zuständige Gericht, das mit der Strafsache befasst ist, seinerseits davon Kenntnis erhält, dass gleichzeitig ein Zivil- oder Familienprozess geführt wird, wird es dem Zivilgericht davon Mitteilung machen, welches sofort seine Unzuständigkeit erklären und die Akten dem zuständigen Gericht vorlegen muss.

Mit Art. 70 des Gesetzes wird die Stelle einer besonderen Staatsanwältin bzw. eines besonderen Staatsanwalts für Fälle von Gewalt gegen Frauen eingerichtet, mit der Aufgabe, die Handlungsrichtlinien der verschiedenen Staatsanwaltschaften im Bezug auf Gewalt im Geschlechterverhältnis zu koordinieren und halbjährlich einen Bericht über geführte Verfahren und über von der Staatsanwaltschaft auf dem Gebiet der Gewalt im Geschlechterverhältnis verfolgte Ansätze zu verfassen (der Bericht wird vom spanischen Generalstaatsanwalt herausgegeben).

Der fünfte Abschnitt enthält weitere wichtige Maßnahmen für den Schutz und die Sicherheit der Opfer. Sie betreffen den Datenschutz und Einschränkungen des Öffentlichkeitsgrundsatzes, Wohnungsverweisung für den Täter, Kontaktverbot, Maßnahmen der Aufhebung des Sorgerechts oder des Besuchsrechts minderjährige Kinder betreffend, waffenrechtlich beschränkende Maßnahmen. 


\section{Ausblick}

Die genannten gesetzlichen Maßnahmen haben nicht das Ergebnis erbracht, welches gewünscht war. Bedauerlicherweise ist die Anzahl der Fälle von Tötungen im Rahmen von Geschlechtergewalt nach wie vor auf einem sehr hohen Niveau. Dabei wird ein hoher Prozentsatz der Opfer durch Immigrantinnen aus Lateinamerika und Nordafrika gestellt.

Im Jahr 2007 wurden 99 Frauen von ihren Ehemännern oder Partnern ermordet. In einer beträchtlichen Anzahl dieser Fälle gab es zuvor keine Anzeigen und keine Schutzanordnungen. Es bleibt notwendig, weitere Anstrengungen zu unternehmen.

Ein wichtiger Schritt ist die gesellschaftliche Ächtung der Gewalt, die sich in erhöhter Anzeigebereitschaft ausdrücken und bewirken könnte, dass in den Prozessen eine größere Anzahl von Zeuginnen und Zeugen zur Verfügung steht, sowie den Opfern mehr Unterstützung beim Ausbrechen aus ihrer Situation gewährt wird. Bei dieser Ächtung spielen die Massenmedien eine wichtige Rolle, die sie mit größerer Sorgfalt ausfüllen müssen.

Gleichzeitig ist es von höchster Bedeutung, die Erziehung zum Gleichstellungsprinzip in der Schule, in den Medien und in den Familien zu verbessern. Nicht zuletzt ist auch die Früherkennung zu nennen, bei der die Gesundheitsberufe und Bildungseinrichtungen eine wichtige Rolle spielen.

Ziel ist das Erreichen eines Wertewandels, der zur gesellschaftlichen Ablehnung der Täter und einer größeren Unterstützung für die Opfer führt. Im heutigen Spanien werden Gewalttaten gegen Frauen zunehmend wahrgenommen und geächtet - insbesondere dank der Bemühungen von Frauenorganisationen im Kampf gegen jegliche Form von Gewalt im Geschlechterverhältnis.

\section{Drei Jahre Gesetz gegen Gewalt im Geschlechterverhältnis}

\section{Thesen und Schlussfolgerungen des Kongresses der Spanischen Juristinnenvereinigung Themis am 30. und 31. Oktober 2008 in Madrid*}

1. Mit diesem Gesetz wird die Gewalt sichtbar gemacht, die sich gegen Frauen als Folge der strukturellen Bedingungen unserer Gesellschaft richtet. Zweifellos ist zutreffend - wie der Generalsekretär der Vereinten Nationen aufgezeigt hat -, dass „sich das Potenzial von Gesetzen gegen Gewalt gegen Frauen nur dann voll ausschöpfen lässt, wenn sie auf effektive Weise angewendet und befolgt werden“, und er fügt hinzu, dass zu diesem Zweck „eine verpflichtende systematische Aus- und Fortbildung in Genderfragen unabdingbar" ist.

In diesem Sinne ist eine Integration der Genderperspektive in die Ausbildungspläne von juristischen und anderen Berufen, die im Prozess der Gewaltbekämpfung intervenieren, unverzichtbar.

2. Von der Regierung wird ein Bewertungsschema gefordert für Verletzungen und Folgeerkrankungen von Opfern von Gewalt im Geschlechterverhältnis, entsprechend den von der WHO beschriebenen Kriterien.

3. Kostenfreie juristische Unterstützung für Opfer von Gewalt im Geschlechterverhältnis muss bei Möglichkeit der freien Auswahl einer Rechtsanwältin/eines Rechtsanwalts umfassend sein, ohne die Opfer im Nachhinein mit dem Kostenrisiko, bei fehlendem Nachweis ihres wirtschaftlichen Unvermögens, zu belasten.

4. Die anwaltliche Vertretung von Geschädigten von Gewalt im Geschlechterverhältnis im Wege der Beiordnung darf nicht durch Personen wahrgenommen werden, die auch Beschuldigte solcher Straftaten vertreten.

5. Rechte und Schutz von Frauen, die sich an das polizeiliche, justizielle oder soziale Unterstützungssystem mit dem Wunsch einer Beendigung ihrer Beziehung zum Täter wenden, müssen garantiert sein.
6. Die an Gerichten angesiedelten Stellen, die mit spezialisiertem Personal unabhängig von den polizeilichen Gefahrenprognosen eigene Gefährdungseinschätzungen vornehmen und dabei die besondere Schwächung des Opfers infolge der erlittenen Gewalt einbeziehen, müssen landesweit eingerichtet werden.

7. Nach einem ersten Schritt nach vorne mit der Anerkennung der grundlegenden Rechte von Frauen ist jedoch eine Gegenbewegung oder ein „Backlash“ zu verzeichnen. Die Bewegung, die eine Abkehr von dem durch die neuen gesetzgeberischen Maßnahmen eingeschlagenen Weg anstrebt, propagiert einerseits neue psycho-juristische Kunstbegriffe, wie das „Syndrom der elterlichen Entfremdung“, „das gemeinsame Sorgerecht ohne Einverständnis“, „das fortbestehende Umgangsrecht von gewaltausübenden Elternteilen" und andererseits die Wiederauferstehung alter sexistischer Mythen wie der Behauptung, dass von Frauen angezeigte Straftaten vorgetäuscht sind.

8. Ein effizienter juristischer und sozialer Schutz von Opfern von Gewalt im Geschlechterverhältnis muss sicherstellen, dass keine Frau, die Anzeige erstattet hat, getötet wird.

\footnotetext{
* Aus dem Spanischen übersetzt von Staatsanwältin Sabine Kräuter-Stockton, Mitglied der Kommission Gewalt gegen Frauen und Kinder des djb; Zugriff auf Originaltext am 16.3.2009: http:// www.mujeresjuristasthemis.org/; Fundación Themis: Conclusiones Congreso Tres Años de la Aplicación de la Ley Integral.
} 